\title{
Calidad de atención en el puerperio inmediato del Hospital Universitario del Valle, Cali, 1993
}

\author{
Lydia Esperanza Miranda Gámez*; Aura F. Pérez Escarlante**; Martha Lucía Vásquez Truissi***
}

\begin{abstract}
RESUMEN: Se realizó un estudio descriptivo-prospectivo con una muestra de 114 mujeres que se observaron desde el ingreso al servicio de puerperio hasta su egreso, para evaluar la calidad de atención que se brinda en el puerperio inmediato en el Hospital Universitario del Valle, Cali, Colombia, durante los meses de octubre a diciembre de 1993. Se evaluaron tres componentes: el científico-técnico que permitió analizar el cumplimiento de normas, la existencia de manuales, recurso humano y material, el de ambiente físico que permitió determinar las condiciones del área en donde se presta la atención y por último el componente interpersonal que permitió identificar la opinión de la usuaria acerca de la atención humanizada recibida. El servicio de puerperio cumplió con el $63.5 \%$ de los requisitos mínimos establecidos para recursos humanos, con un $68.3 \%$ para recursos materiales, con un $18.2 \%$ para el cumplimiento de normas y un $66.6 \%$ para la existencia de manuales. El $\mathbf{6 8 . 8 \%}$ de las mujeres opinó que la atención recibida fue humanizada, las condiciones del ambiente físico obtuvo un $56.7 \%$ de cumplimiento de los requisitos mínimos establecidos. Estos resultados reportaron que ningún componente obtuvo un porcentaje mayor del $80 \%$ (porcentaje mínimo establecido), lo que indicó que la calidad de atención en el puerperio inmediato no fue adecuada.
\end{abstract}

PALABRAS CLA VES: Calidad de Atención, calidad de servicio, evaluación de la calidad.

SUMMARY: This is a descriptive-prospective study to evaluate the quality of the medical attention during the immediate puerperium at the Hospital Universitario del Valle in Cali, Colombia, on a sample of 114 women observed from october to december 1993. Three components were evaluated: the scientific-technic one, that allowed the analysis of the fulfillment of the norms, the existence of manuals and the human and material resources; the physical environment that allowed to determine the condition of the area where the attention is offered; and finally the interpersonnel component to know the opinion of the patient about the humanized attention received. The puerperium service fulfilled the $63.5 \%$ of the minimal requisites established for human resources; the $68.3 \%$ for material resources; the 18.2 for fulfillment of the norms and the $66.6 \%$ for the existence of manuals. $68.8 \%$ of the women reported that the medical attention was humanized; the conditions of the physical environment obtained the $56.7 \%$ of the established minimal requisites. The results showed that any component obtaneid more than $80 \%$ (minimal established percentage) indicating that the quality of the attention in the immediate puerperium was inadequate.

KEY WORDS: Attention quality, service quality, quality evaluation.

\section{Introducción}

El sistema de salud tiene como finalidad proporcionar a la población una atención con servicios oportunos, suficientes, a bajo costo y de buena calidad. Con este propósito se desarrollan programas de atención a las personas y al medio ambiente. Entre los primeros se encuentra el programa de atención al grupo maternoinfantil, el cual es prioritario en las políticas de salud en los países latinoamericanos.

La morbilidad y mortalidad de este grupo sirven como indicadores para evaluar la calidad de atención, por ello es necesario valorar periódicamente sus componentes para determinar el cumplimiento de metas establecidas para la atención de la madre y recién nacido.

\footnotetext{
* Jefe de la División de Desarrollo y Comportamiento Humano. Instituto Departamental de Salud de Nariño. Pasto, Colombia.

** Enfermera de Sala de Partos y Area Quirúrgica. Hospital Materno Infantil del Este-Petare. Dr. Joel Valencia Parpacen. Caracas, Venezuela.

*** Profesora Titular de la Escuela de Enfermería, Facultad de Salud, Universidad del Valle, Cali, Colombia.
}

La Organización Mundial de la Salud estima que cada año mueren 500.000 mujeres en los países en desarrollo como consecuencia de las complicaciones del embarazo, parto y puerperio (1). Esta cifra se considera alarmante sobre todo si se tiene en cuenta que la mayoría son muertes evitables mediante una atención oportuna y de buena calidad.

En Colombia la mortalidad materna fue de 110 muertes por 100.000 nacidos vivos en 1990 , estimándose un elevado subregistro lo cual amerita investigaciones en profundidad para constatar esta información. En Cali la mortalidad materna en 1992 fue de 80 por 100.000 nacidos vivos (2), las causas de estas muertes son las mismas que para el resto del país.

En tal sentido, las políticas para la reducción y control de la morbilidad y mortalidad materna deben tender a fortalecer la infraestructura de los servicios y a enfatizar en una atención más humana y de mejor calidad; ofreciendo servicios eficientes, eficaces y equitativos.

Estudios sobre la calidad de atención en los servicios de salud (3-6) confirman la necesidad de evaluarla, con 
la finalidad de establecer las medidas correctivas adecuadas y así mejorar la atención.

La evaluación de la calidad de atención en la población materno infantil ha sido considerada como prioridad ya que tiende a mejorar las condiciones de eficiencia, eficacia y equidad de la atención (7).

En Cali se realizó un estudio sobre calidad de atención del parto y postparto en el Centro Hospital Joaquín Paz Borrero en 1991 (8), el cual reportó que el control del puerperio inmediato y mediato fue inadecuado en un $100 \%$.

El presente estudio descriptivo-prospectivo se realizó en el Hospital Universitario del Valle (H.U.V.) de la ciudad de Cali, Colombia de octubre a diciembre de 1993 con el objeto de evaluar la calidad de atención en el puerperio inmediato que abarcaba 3 componentes: el científico técnico, el interpersonal y del ambiente físico.

\section{Materiales y métodos}

Se diseñó un estudio descriptivo para el cual se calculó el tamaño de la muestra con base en la siguiente fórmula:

$$
\begin{gathered}
\mathrm{n}=\frac{\mathrm{n}_{\mathrm{o}}}{1+\frac{\mathrm{n}_{\mathrm{o}}}{\mathrm{N}}} \\
\mathrm{n}_{\mathrm{o}}=\frac{\mathrm{z}^{2}}{\mathrm{~d}^{2}} \mathrm{p \times q}
\end{gathered}
$$

donde:

$\mathrm{N}=477$ (promedio de número de nacimientos atendidos en el período de enero a marzo de 1993 en el H.U.V. de la ciudad de Cali).

$\mathrm{z}=1,96$

$\mathrm{d}=5 \%$

$\mathrm{p}=89 \%$ (prevalencia de parto institucional en Cali, en 1992)

$\mathrm{q}=11 \%$

$\mathrm{n}=114$

Se estudiaron 114 mujeres que se encontraban en el servicio de puerperio del H.U.V., cuyo parto fue atendido en la misma institución.

Las mujeres estuvieron distribuidas en los siguientes grupos de edad: $14-17$ años: 20 (17.5\%), 18-34 años: 79 (69.3\%) y 35 y más años: 15 (13.2\%).

La unión libre fue el estado civil predominante $(60.5 \%)$ seguido de solteras $(21.2 \%)$ y por último las casadas (18.4\%). El nivel de escolaridad fue $44.7 \%$ con secundaria incompleta, $21.9 \%$ primaria incompleta, $16.7 \%$ primaria completa, $10.5 \%$ secundaria completa, analfabetas $5.3 \%$ y otros estudios $0.9 \%$. El $71 \%$ procedían de Cali, el $14.9 \%$ de área rural y $14 \%$ de municipios fuera de Cali. Del $71 \%$ procedente de Cali el $26.3 \%$ pertenecían al estrato bajo-bajo, el $25.4 \%$ al estrato bajo, y el $19.3 \%$ al estrato medio bajo.
La recolección de los datos se efectuó en el período comprendido entre octubre a diciembre de 1993. La selección de mujeres se hizo por conveniencia, hasta completar el tamaño de la muestra, la observación se llevó a cabo desde el ingreso de la usuaria al servicio del puerperio hasta que fue dada de alta.

La observación estructurada y el cuestionario fueron los métodos de recolección de información. Para determinar el cumplimiento de las normas en la atención dada por el personal de salud a la usuaria se utilizó una lista de chequeo con base en las normas de atención materna establecidas por el Ministerio de Salud (9), en el instrumento utilizado por otro estudio similar (10) y las sugeridas por expertos del tercer nivel de atención (11).

Para obtener información de las usuarias acerca de la percepción de ellas con respecto a la atención humanizada brindada, se elaboró un formulario y se aplicó a la usuaria antes de ser dada de alta. Para evaluar los recursos humanos, materiales, ambiente físico y existencia de manuales se diseñó una guía basada en las pautas del Centro Latinoamericano de Perinatología (CLAP), y las propuestas en el documento sobre condiciones de eficiencia de los servicios Maternos Infantiles en el Departamento del Valle, Colombia, 1985 (12).

Se tomó como criterio de cumplimiento de cada uno de los componentes de calidad de atención, la obtención de $100 \%$ del puntaje total asignado a cada uno de los indicadores. Se estableció como calidad adecuada a aquel componente que obtuvo al menos el $80 \%$ de cumplimiento.

\section{Resultados}

\section{Componente científico técnico}

El cumplimiento de las normas establecidas para la atención a la madre y a su hijo durante el puerperio fue el siguiente:

La revisión de historia clínica se cumplió en el 100\% de las usuarias.

La toma de signos vitales no se cumplió en la totalidad de las mujeres observadas. Al relacionar el cumplimiento de la norma con el índice ocupacional, cuando fue igual o menor al $100 \%$, la norma no se cumplió en un $75 \%$ y cuando el índice ocupacional era mayor al $100 \%$ la norma no se cumplió en un $25 \%$.

La toma de temperatura se cumplió en el $87.7 \%$ de la muestra. La toma de la frecuencia cardíaca no se cumplió en el $100 \%$ de las mujeres de la muestra.

La vigilancia de involución uterina se cumplió en el $28.9 \%$ de la muestra y el registro de las actividades en un $74.6 \%$ de la muestra.

La vigilancia de la eliminación urinaria se cumplió en el $49.1 \%$, de las 69 mujeres a quienes se les preguntó si eliminaron, se registró la actividad a $56(81.1 \%)$ y de las $45(39.5 \%)$ a quienes no se les preguntó se registró la actividad como realizada a 4 mujeres $(8.9 \%)$.

A 82 mujeres se les realizó episiorrafia, y sólo al $8.5 \%$ se les practicó todos los cuidados de la misma y se registró la actividad en un $18.3 \%$.

De las 114 mujeres observadas, $102(89.5 \%)$ tuvieron recién nacidos vivos y fueron trasladados juntos con su 
madre a la sala. La norma cuidado del recién nacido con el total de actividades no se cumplió en el 100\%. Las actividades se realizaron así: se confirmó el traslado del recién nacido con su madre al $95.1 \%$ y se registró al $61.8 \%$. La orientación a la madre en lactancia materna se cumplió en un $6.9 \%$. El cuidado del muñón umbilical se cumplió en un $2.9 \%$; se registró en un $13.7 \%$. Baño del recién nacido se llevó a cabo en un $15.7 \%$ de los niños observados y se registró la actividad al $31.4 \%$.

La vigilancia de la eliminación urinaria al recién nacido se realizó en el $19.6 \%$ de los niños observados. La vigilancia de la frecuencia respiratoria se cumplió en el $31.3 \%$ de los recién nacidos observados, y se registró frecuencia respiratoria al $40.1 \%$.

La ambulación temprana de la usuaria con la totalidad de sus actividades se cumplió en el $1.8 \%$ de la muestra y se registró la actividad al $11.4 \%$.

La información a la usuaria sobre cuidados durante el postparto, el baño diario, el aseo perineal, la alimentación balanceada y la importancia de la cita postparto se ofreció completa al $5.3 \%$ de las usuarias. La información a los familiares se cumplió en un $6.1 \%$.

La orientación sobre métodos de planificación familiar se cumplió en un $42.1 \%$.

El cumplimiento de la aplicación de vacunas fue del 9.8\%. La norma de examen físico se cumplió en un $56.1 \%$ de la población observada: el examen físico del recién nacido se cumplió en un $94.1 \%$ y el examen físico de la usuaria en un $60.5 \%$.

La entrega de documentos: carné materno, registro y entrega del carné infantil y entrega del certificado de nacimiento, no se cumplieron en la totalidad de la muestra.

En resumen, el porcentaje total de cumplimiento de normas fue de $18.2 \%$ (gráfico 1 ).

De acuerdo con las condiciones de eficiencia de los servicios Materno Infantiles en el departamento del Valle del Cauca y las pautas del CLAP se encontró que: el servicio de puerperio cuenta con manual de normas y funciones pero no existe manual de procedimientos.

En cuanto a recursos humanos, el servicio de puerperio cumple con un $63.5 \%$ de los requisitos mínimos para su buen funcionamiento.

Discriminando lo relacionado a estos recursos se obtuvo un cumplimiento de la norma en un $90 \%$ en el ítem de médico jefe. El $50 \%$ en los criterios establecidos para médicos. En este sentido se observó que no había disponibilidad de médico obstetra, ni médico anestesiólogo las 24 horas del día. El médico pediatra si estaba las 24 horas de turno pero no era exclusivo del servicio de puerperio.

Para el personal de enfermería el cumplimiento fue del $40.3 \%$ a este respecto se observó: ausencia de enfermera subjefe para hospitalización con postgrado en salud pública o curso equivalente. No se cumple la relación de 8 horas enfermera jefe de puerperio por 24 camas (sábados, domingos y feriados, hay una enfermera jefe por tres (3) servicios), existe una enfermera jefe por cada 300 e gresos (exceso de 150), no existe en el servicio una enfermera jefe especializada en la atención del recién nacido y prematuros; en cuanto a personal auxiliar
Gráfico 1

DISTRIBUCION PORCENTUAL DEL CUMPLIMIENTO DE NORMAS EN EL SERVICIO DE PUERPERIO

Hospital Universitario del Valle - Cali, Oct-Dic. 1993

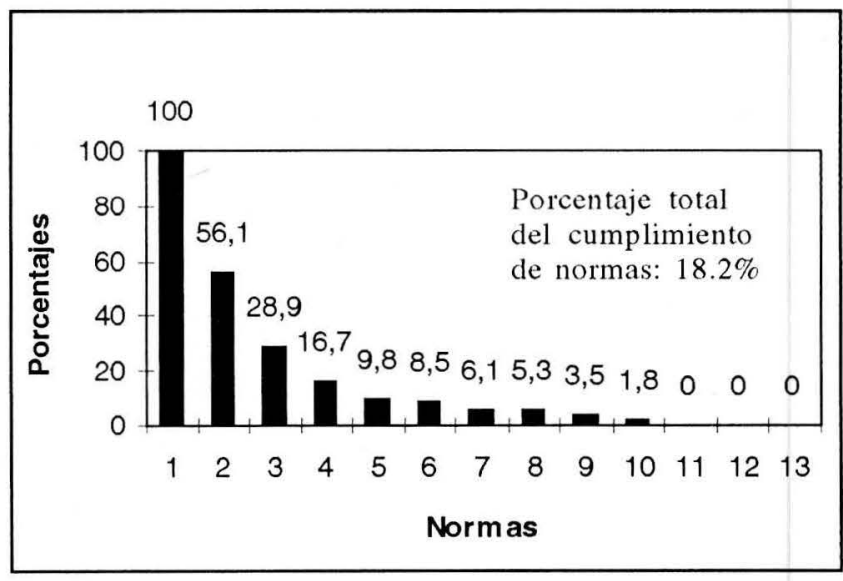

Convenciones

1. Revisión historia clínica

2. Examen físico

3. Vigila involución uterina

4. Vigila eliminación y deposición

5. Aplicación de vacunas.

6. Cuidados de episiorrafia

7. Informa a los familiares

8. Informa y orienta a la puérpera

9. Orienta a la madre

10. Propicia la ambulación temprana

11. Toma de signos vitales

12. Cuidados del recién nacido

13. Entrega de documentos

de enfermería el servicio presentó un $64.2 \%$ de cumplimiento, cuenta con más de dos (2) auxiliares bajo la supervisión de una enfermera jefe; para el ítem de personal de trabajo social, se obtuvo un cumplimiento del $100 \%$, la secretaria del servicio cuenta con nivel secundario y curso de secretariado, obteniendo el $100 \%$ de cumplimiento para el buen funcionamiento del servicio; se carece de una persona de servicios generales, por cada 30 camas en 24 horas.

En la norma de recursos materiales el servicio de puerperio cuenta con el $93.3 \%$ de elementos de consumo (42 puntos), y con el $43 \%$ de material devolutivo (26 puntos), obteniéndose un porcentaje promedio de $68.3 \%$ de cumplimiento de lo establecido.

Con referencia al componente científico-técnico, se encontró que el servicio de puerperio tiene el $54.1 \%$ de las características requeridas para una adecuada atención (gráficos 2 y 3).

\section{Ambiente físico}

La sala de puerperio consta de cuatro (4) cubículos, los cuales tienen ocho (8) camas cada uno; hay una cama de puerperio por cada 30 camas de hospitalización, lo que permite establecer que el servicio cumple con el 
$83.3 \%$ de los requisitos para una adecuada atención. Uno de los cubículos es utilizado para pacientes de alto riesgo obstétrico.

$$
\text { Gráfico } 2
$$

COMPONENTE CIENTIFICO-TECNICO EN PUERPERIO INMEDIATO

Hospital Universitario del Valle - Cali, Oct-Dic. 1993

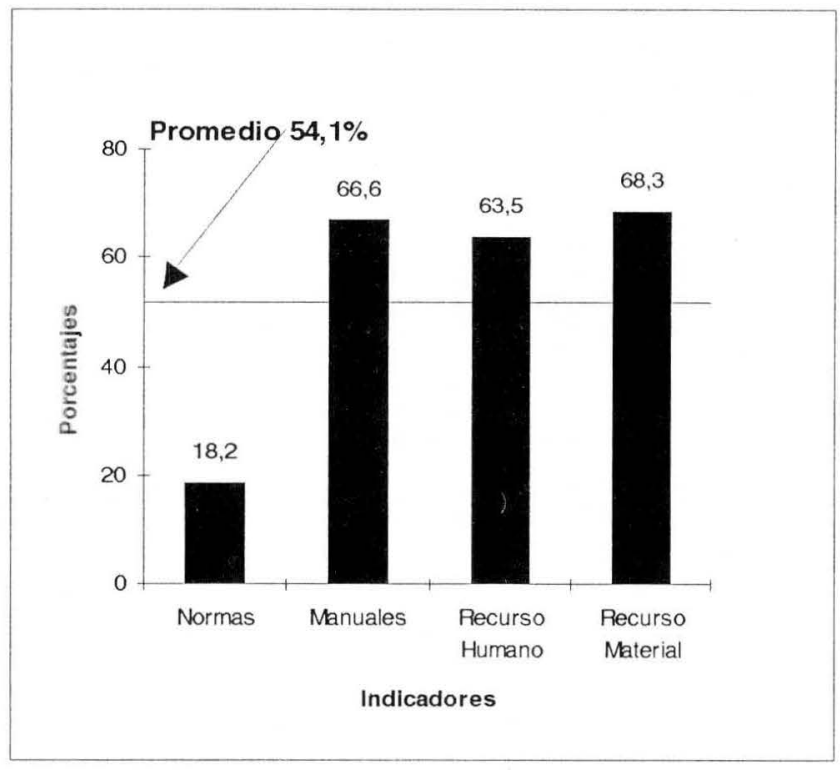

El ítem: sanitario, limpieza y confort en el servicio de puerperio obtuvo un $30 \%$ de cumplimiento de los requisitos establecidos para un buen funcionamiento. Las deficiencias fueron: los pisos y paredes no son lavables ni de material inalterable, no existe un baño completo por cada 20 miembros del personal, ni de un sistema adecuado de ventilación, ni un baño completo por cada 10 camas de hospitalización, ni biombos o cortinas para respetar la intimidad de la paciente al momento de realizar los procedimientos.

Los anteriores datos permiten establecer que el porcentaje promedio de cumplimiento de los requisitos de este componente es de $56.7 \%$ (gráfico 3 ).

Atención humanizada (Componente interpesonal)

De las mujeres que expresaron que fueron informadas sobre su estado de salud, $(56,2 \%)$ a 20 de ellas no se le realizó esta actividad $(31.25 \%)$ y de las 50 mujeres que manifestaron no haber recibido información (43,8\%) a 6 de ellas (12\%) se les realizó esta actividad.

El 69.3\% de las mujeres manifestaron haber recibido información acerca de la salud de su recién nacido, al $22.8 \%$ (18 madres) no se les realizó esta actividad, de las 35 madres que dijeron no haber recibido información, a $11(31.42 \%)$ se les realizó esta actividad. Estos resultados se verificaron en la observación. De las 114 mujeres encuestadas, el $47.4 \%$ manifestó que fueron informadas sobre los procedimientos a realizarles, al $41.2 \%$ se les explicó cada uno de los procedimientos según lo expresado por las usuarias. Con relación al trato el $70.2 \%$ indicó que era bueno; el $20.2 \%$ regular; el $7 \%$ excelente y el $2.6 \%$ malo.

El $66.7 \%$ de las madres refirieron no haberse sentido incómodas en la sala de puerperio.

De las 114 mujeres encuestadas, el $19.3 \%$ expresó no haber solicitado ayuda en la sala de puerperio, el $80.7 \%$ solicitó ayuda en el puerperio y se le brindó ayuda oportunamente al $68.4 \%$.

La habilidad del personal le infundió seguridad en los procedimientos realizados a $86.6 \%$ de las mujeres encuestadas. E1 $94.7 \%$ de las madres manifestaron que se sintieron respetadas en la sala.

Al 48.2\% de las mujeres las llamaron por su nombre, con las demás $(50.8 \%)$ se usaron expresiones como "gordita", "señora", "la paciente de la cama..., entre otras.

De las 114 mujeres, el $65.8 \%$ consideró como buena la atención recibida desde el momento que salió de la sala de partos; el $19.3 \%$ consideró que fue regular, el $12.3 \%$ excelente y $2.6 \%$ la clasificó como mala.

En resumen, el porcentaje total de opiniones que indican la calidad de atención humanizada brindada fue del $68.8 \%$ (gráfico 3).

Gráfico 3

COMPONENTES DE LA CALIDAD DE ATENCION EN PUERPERIO INMEDIATO

Hospital Universitario del Valle - Cali, Oct-Dic. 1993

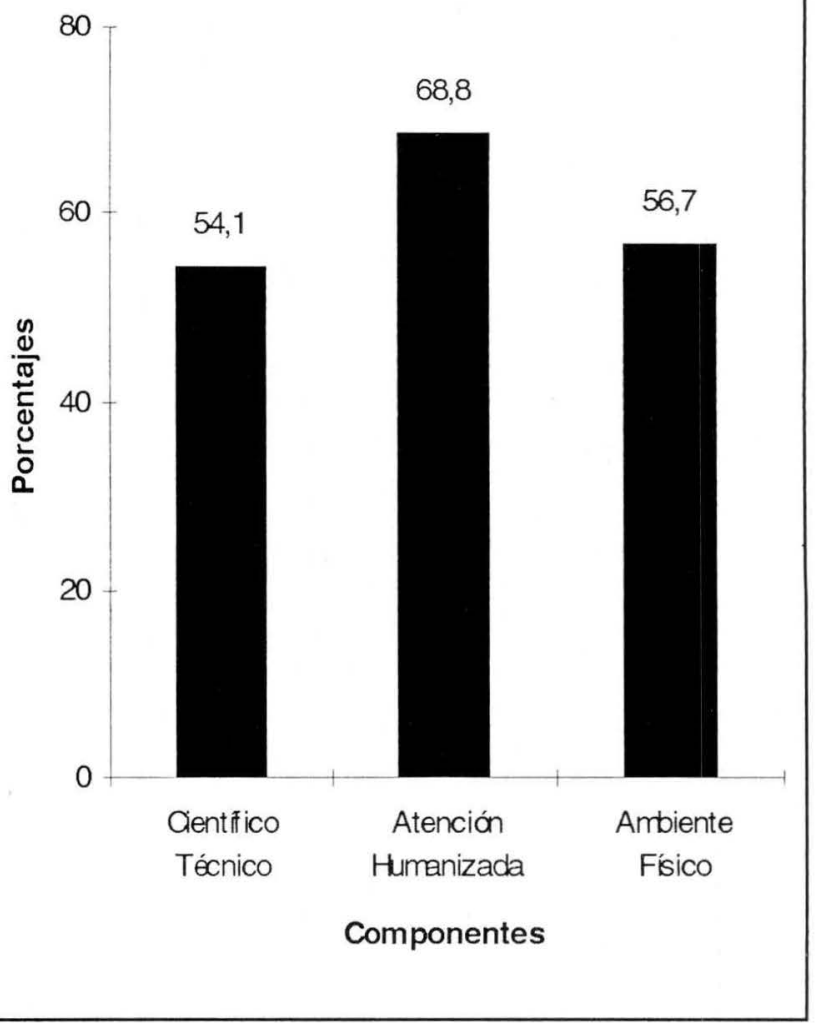




\section{Discusión}

Dentro de la calidad de atención se contempla el cumplimiento de las normas como un indicador para obtenerla. Los resultados del estudio evidenciaron que el incumplimiento de las normas no coincidió con los momentos en que el servicio estaba con un índice ocupacional mayor o igual al $100 \%$, lo que puede indicar que esto no fue un factor contribuyente en la situación encontrada. El incumplimiento de las normas afecta la calidad de atención, puesto que no permite detectar oportunamente las complicaciones de la puérpera, situación similar se reportó en el estudio calidad de atención del parto y postparto (13).

La observación estructurada permitió establecer que el porcentaje promedio de cumplimiento del registro de las actividades fue bajo, (40\%), se registraron actividades no realizadas, coincidiendo con los resultados obtenidos en el estudio anteriormente mencionado, lo que puede indicar que hay falta de supervisión, y una concientización en la responsabilidad ética del personal que labora en el servicio, lo cual contribuye a garantizar la atención integral y continua a la usuaria. El registro de las actividades de cada norma es un mecanismo necesario para verificar su cumplimiento y brindar así los datos indispensables para el seguimiento, la atención integral por el equipo de salud y su evaluación posterior (14).

La supervisión por los jefes inmediatos al personal médico y de enfermería contribuye a asegurar el cumplimiento correcto de la norma en todos los casos (15), situación que no se cumple en este servicio si se tiene en cuenta que no hay enfermera jefe, ni, médico obstetra disponible en las 24 horas del día.

Los resultados reportados por esta investigación muestran un bajo porcentaje en el cumplimiento de la norma sobre orientación y aplicación de vacunas contrario a lo encontrado por otro estudio similar (16). La Secretaría de Salud Municipal suministró y aplicó la vacuna a los niños en los cuales se cumplió la norma. La actividad de aplicación de vacuna no se lleva a cabo en el servicio debido a que el hospital no cuenta con central de conservación de los biológicos, esto hace que no se aproveche el contacto del recién nacido con el sistema de salud para iniciar el esquema de vacunación.

El fomento del vínculo psicoafectivo de la pareja y familiares con el recién nacido es una de las normas que debe tener en cuenta el personal de salud en la atención humanizada. La observación permitió establecer que las deficiencias en ésta última actividad, no se debieron a reglas que impidieran la visita de los familiares a las mujeres. Valdría la pena analizar las causas que propiciaron el hecho de que el personal que trabaja en el servicio no fomenta este vínculo como parte de la atención humanizada.

La existencia de manuales que contengan las normas, procedimientos y funciones permite establecer canales de operación que aseguran en parte la calidad de atención, sin embargo si estos manuales "no se difunden, adecúan, evalúan y actualizan con la participación de todos los niveles del sistema de servicios; solamente se obtiene un documento escrito ignorado por la mayoría, mientras la atención se brinda según criterios individuales o de grupos, siguiendo tradiciones o avances tecnológicos adoptados indiscriminadamente" (17), afectando la calidad de atención, más aún, si sólo se cuenta con el $66 \%$ de los manuales necesarios.

El recurso humano es el componente esencial para llevar a cabo la atención a la mujer. La escasez de personal lleva a la delegación de actividades y a muy poca supervisión o a deteriorar las relaciones interpersonales con la usuaria. La falta de personal capacitado asignado al área pone en peligro una atención de óptima calidad.

La satisfacción de la usuaria es una medida fundamental de la calidad de atención, porque proporciona información sobre el éxito del proveedor en alcanzar las expectativas de ella. La opinión de las usuarias, relacionada a la atención humanizada recibida en el estudio reportó bajos y altos porcentajes en las respuestas a las preguntas sobre este tema. La dualidad de estos resultados y las opiniones expresadas por las madres en los ítems que hacen referencia a la incomodidad en la sala y aspectos que más les llama la atención y les gusta, indican que a pesar de encontrarse problemas de relaciones interpersonales entre la usuaria y proveedor, las mujeres manifiestan recibir atención humanizada.

Hubo factores que influyeron en las respuestas dadas por las madres:

a) El momento en que se realizó la encuesta debido a que se encontraban en fase de adaptación a la maternidad, caracterizada por la pasividad y preocupación o por sus propias necesidades; b) El lugar de obtención de la información, debido a que las usuarias evitan criticar a quienes prestan la atención estando todavía hospitalizadas; c) Algunas características del grupo encuestado como la edad, el estrato socioeconómico y la escolaridad (18), los cuales pudieron influir en la opinión sobre los servicios recibidos puesto que la mujer desconoce los aspectos científicos y los derechos que tiene como persona la usuaria de un servicio (19-20).

La deficiencia de las condiciones de ambiente físico impide el adecuado funcionamiento del sistema de atención. Estas deben estar presentes en concordancia con las necesidades de cada servicio e institución (21). Situación que no se da en el servicio de puerperio.

Las deficiencias en el servicio de puerperio podrían ser el reflejo de problemas graves a nivel gerencial, organizacional, financiero y de la satisfacción del personal que presta la atención. Para mejorarla se hace necesario realizar reestructuraciones con base en las deficiencias detectadas, las que deben ser sometidas a evaluaciones periódicas para garantizar una adecuada calidad de atención a la madre y al recién nacido.

La búsqueda de la calidad se apoya en dos ideas claves: el reconocimiento por parte de cada profesional, de su capacidad para contribuir a la mejora de la calidad del servicio que presta y la introducción de medidas que hagan posible convertir en dinámico lo que parece estático. 


\section{BIBLIOGRAFIA}

1. Arias M., Palacios F. Situación materno-infantil a nivel nacional, regional y local. Universidad de Antioquia. Medellín, 1987; 35A.

2. Secretaría de Salud Municipal. Oficina de Epidemiología. Informe mortalidad materna. Cali, 1992.

3. Nine S., Bayes K., Christian S., Dillon B. Organizing Quality Assurance in a Maternal child Health División. JOGN. January-February. 1992; 21(1): 28-32

4. Aguirre-Gas H. Evaluación de la calidad médica. Expectativa de los pacientes y de los trabajadores de las unidades médicas. Salud Pública México. 32: 170-180.

5. Castrillón M., Escobar I., Pulido S. Calidad de atención en sala de trabajo de parto, instituciones oficiales de salud. Antioquia. Universidad de Antioquia. Medellín, 1990; 23-26.

6. Chong X., Noreña C. Eficacia del Subprograma de atención al parto y postparto del Centro Hospital Joaquín Paz Borrero. Universidad del Valle. Cali, 1991; 77-80.

7. Rico J y Col. Condiciones de eficiencia de los servicios maternoinfantiles del Departamento del valle, Colombia, Universidad del Valle. 1985; 220-221.

8. Chong Noreña. Op. cit. pp. 58-60.

9. República de Colombia. Ministerio de Salud. División Materno Infantil. Atención Materna y Perinatal con criterio de riesgo. 1a. Ed. Santa Fe de Bogotá. 1988.
10. Chong, Noreña. Op. cit. p. 116.

11. Delgado C. Normas generales y específicas sobre la atención hospitalaria de la madre y el recién nacido. Hospital Universitario del Valle. Cali, 1987; 17.

12. Rico J y Col. Condiciones de eficiencia de los servicios maternos infantiles del Departamento del Valle. pp. 251-265.

13. Chong X., Noreña C., Vásquez M. Calidad de atención en el parto y postparto del Centro Hospital Joaquín Paz Borrero. Cali, 1991. Colombia médica. vol. 25 No. 1. Cali, 1994. p. 6.

14. República de Colombia. Atención materna y perinatal. Op. cit. 31.

15. Salud Perinatal. Boletín del CLAP. Op. cit. p. 54

16. Chong X., Noreña C., Vásquez M. Calidad de atención en el subprograma de parto y postparto. Op. cit. pp. 6-7.

17. Salud perinatal. Boletín del CLAP. Op. cit. p. 52.

18. Holsclaw P. Conceptos Básicos de enfermería. Diana Edit. Primera México $1982 ; 25$.

19. Donabedian A. La calidad de la Atención Médica. La prensa Médica Mexicana. S.A. Edit. Michigan, 1983; pp. 3-7.

20. Holsclaw. Op. cit. p. 25.

21. Pabón. Evaluación de los servicios de salud. Op. cit. p. 136.

\section{EVENTOS}

I CONGRESO I.ATINOA MERIC A NO DE MASTOL OGIA

Centro de Convenciones Gonzalo Jimenez de Quesada del 18 al 22 de jullo de 1995.

Motel Tequendama Samafé de Bogota 28, 29 y 30 de jullo de 1995

Informes: 6162970

\section{CONGRESO INTERNACIONAL DE TIRTII IDAD Y ESTERII IDAD Montpellier Irancia}

17 al 22 de septlembre de 1995

\section{CONGRESO COLOMBIANO DE GINECOI. OGIA ONCOI OMICA}

Call, Noviembre 1, 2 y 3 de 1995

Infornes: lnstituto Nacional de Cancerologta

Santa Fe de Bogotá. Telefono: 2335623

\section{FE DE FRRATAS}

En la pagina 70 de la Revista No. 1 del presente volumen, donde dice METOL.AX ANTE debe decir METROTEXATE. 